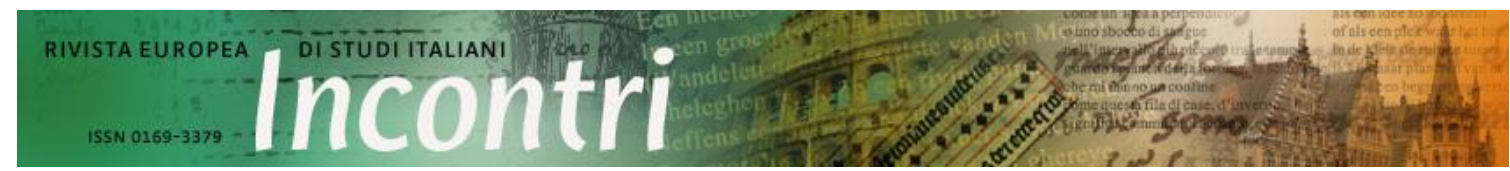

Anno 35, 2020 / Fascicolo 2 / p. 69-81 - www.rivista-incontri.nl - http://doi.org/10.18352/incontri.10353 (c) The author(s) - Content is licensed under a Creative Commons Attribution 3.0 Unported License Publisher: Werkgroep Italië Studies, supported by Utrecht University Library Open Access Journals

\title{
Commemorare vittime di violenza La street art come luogo di resistenza e discorso di positività
}

\author{
Inge Lanslots, Paul Sambre \& Eliana Maestri
}

\begin{abstract}
Se chiedersi a chi appartengano le città non è mai stato più difficile, forse è il momento di rinunciare alle facili risposte. E di lasciare che anche i muri dicano la loro. ${ }^{1}$
\end{abstract}

Il 23 maggio 2017, nell'ambito di una manifestazione commemorativa a Capaci, parenti di vittime della mafia ridipinsero il murales 'no mafia'. La versione originale della scritta risaliva a pochi giorni dalla strage del 1992, che uccise il giudice Giovanni Falcone, la moglie Francesca Morvillo e i tre agenti di scorta, Antonio Montinari, Vito Schifani e Rocco Dicillo, ma fu ben presto cancellata per appropriati set cinematografici di film e fiction sulla strage di Capaci. L'attuale scritta copre un muro del casolare Amap situato su una collina da cui fu azionato il telecomando della bomba che fece saltare in aria l'autostrada che porta a Palermo per colpire uno dei maggiori simboli della lotta alla mafia. Dopo le prime cancellazioni, la scritta risorse nel 2005 e nel giro di dodici anni fu ripristinata più volte, nonostante il carattere illegale dell'opera. Dal 2005 a ora la scritta, sempre illegale, persiste ed è tollerata da vari attori sociali, incluse le istituzioni. In un certo qual senso il murales può essere considerato un palinsesto positivo nella vasta produzione della street art, ${ }^{2}$ la cui conservazione si deve anche all'Amap stessa che nel 2015, al fine di mantenere inalterato il messaggio antimafia della scritta, fece cancellare una frase sottostante a 'no mafia'. ${ }^{3}$

Un anno prima dell'ultimo restauro del murales a Capaci, a Roma, invece, furono cancellate le opere murarie dedicate alla memoria di Carlo Giuliani, morto nel 2001 durante il G8 tenutosi a Genova. Il Municipio VIII, in qualità di unità amministrativa, fece anche cancellare la scritta muraria dal testo della canzone del gruppo hip hop romano antiviolenza Assalti Frontali 'Non spegni il sole se gli spari addosso' dipinta in riferimento alla morte di Giuliani, cancellazione a nome del 'decoro romano', un

\footnotetext{
${ }^{1}$ I. Salvi, 'Muri e memoria: la guerra del decoro che ingrigisce le città', in: CORE. Circuiti Organizzati Resistenze Editoriali, 15 ottobre 2016, https://www.csoalastrada.net/core-online/dispacci/muro-carlogiuliani/ (20 aprile 2020).

${ }^{2}$ H. Kenaan, 'Streetography: on Visual Resistance', in: Journal of Aesthetics and Phenomenology, 3, 2 (2016), p.155.

${ }^{3}$ Cfr. 'Capaci, ripulita dalla scritta "oltraggiosa" la "casina no mafia"', in: PALERMOTODAY, 12 novembre 2015, https://www.palermotoday.it/cronaca/ripulita-scritta-capaci-casolare-no-mafia.html (20 agosto 2020); A. Noto, 'Vandalizzata la casetta “No Mafia”. L'anno scorso fu la volta della stele Falcone', in: il Giornale di Isola, 10 novembre 2015, http://www.giornaleisola.it/archives/5215 (20 agosto 2020).
} 
criterio non spiegato ulteriormente dalle autorità locali ma riguardante chiaramente l'organizzazione estetica dello spazio urbano. Così scomparve anche il dipinto del volto del giovane Giuliani situato in fondo alla scalinata monumentale di piazza Brin del quartiere Garbatella. Ma nell'ottobre dello stesso anno, durante la manifestazione 'DeCore: i nostri muri parlano', ${ }^{4}$ spuntarono opere murarie ex novo del ritratto di Giuliani circondato dalle date di nascita e di morte, come se il murales fosse la parte superiore di una tomba funeraria che ormai dialoga con slogan e altre opere grafiche sui muri adiacenti.

Da questo sunto fin troppo sbrigativo della genesi dei due murales (a Capaci e alla Garbatella), ambedue espressioni visive di pratiche commemorative illecite, definite ribelli e trasgressive dagli autori stessi, si evidenzia quanto siano complessi i rapporti che si intessono a partire dalla street art. In tale luce, il presente contributo parte da un confronto dettagliato della genesi dei murales summenzionati per mettere a fuoco la complessità dei rapporti che si intrecciano a partire dalla commemorazione di vittime di violenza e/o di criminalità, soffermandosi innanzitutto sulla specificità dello spazio urbano circostante e sul ruolo variegato degli attori sociali coinvolti. Il confronto, dall'approccio interdisciplinare, prende spunto dai memory studies, campo già di per sé interdisciplinare, ma si riferirà anche a nozioni tratte dalla sociologia e dagli studi di visual culture ${ }^{5}$ in quanto essi offrono il quadro interpretativo idoneo per corroborare la tesi avanzata da Luca Palermo secondo la quale la street art si iscrive non solo nel dibattito pubblico ma anche nell'attivismo civico. Come afferma lo stesso Palermo, l'attivismo contro la violenza criminale crea nuovi spazi pubblici che vanno oltre quelli fisici:

Street art is not just art that uses public space, but a type of art that almost corresponds to this space, is a distinctive feature of it, makes it special and welcoming to its users, and takes on an instructional and social role in relation to an increasingly wider public. ${ }^{6}$

Tra le caratteristiche dello spazio pubblico attorno ai murales in effetti si nota una tensione che si iscrive in una lunga serie di memorie divise tipiche della storia dell'Italia contemporanea. Nell'ambito dei memory studies, che dalla fine del secolo scorso mettono in risalto quanto sia problematico il rapporto con il passato recente, ${ }^{7}$ lo storico John Foot sostiene che: '[d]ivided memories created memoryscapes and

\footnotetext{
${ }^{4}$ Furono coinvolti gli attivisti di Casetta Rossa, il centro sociale La Strada e la comunità della Garbatella. Cfr. 'Roma, ripristinato il murale di Carlo Giuliani alla Garbatella', 16 ottobre 2016, https://roma.repubblica.it/cronaca/2016/10/16/foto/roma_ripristinato_il_murale_di_carlo_giuliani_all a_garbatella-149922043/1/\#1 (20 aprile 2020).

${ }^{5}$ La presente analisi è nata nell'ambito di un progetto dedicato alla street art, intitolato 'Engaging in Cities through Mural Visual Street Art', una collaborazione tra Eliana Maestri (University of Exeter), Inge Lanslots e Paul Sambre (KU Leuven), iniziato con i fondi Europe Network Fund erogati dall'Università di Exeter nell'ambito dei progetti di Global Partnerships e che verrà continuato con finanziamento di followup.

A questo scopo i ricercatori, che hanno svolto fieldwork dal maggio 2019 al febbraio 2020 nelle città di Bruxelles, Anversa, Londra, Palermo e Roma, desiderano esprimere la loro gratitudine a tutte le guide, gli esperti, gli artisti e altri attori coinvolti nella street art, in particolare Antonio Vassallo e i fondatori delle due associazioni di Addiopizzo (cfr. infra), nonché Fabio Barilari, architetto, illustratore e studioso, che per C-Rome organizza visite guidate a quartieri poco esplorati dal pubblico (https://crome.com/urban-walks-of-rome-art-and-architecture/, 10 novembre 2020).

${ }^{6}$ L. Palermo, 'Beyond the Wall: Street Art in the Fight against the Camorra', in: Modern Italy, 22, 4 (2017), p. 382.

7 Si vedano: A. Assman \& S. Conrad (eds.), A Memory in a global age: discourses, practices and trajectories, Basingstoke, Palgrave Macmillan, 2010; A. Erll \& A. Nünning (eds.), A Companion to Cultural Memory Studies, Berlin, De Gruyter, 2010; A. Erll, Memory in Culture, New York, Palgrave Macmillan, 2011; M. Rothberg, Multidirectional memory: remembering the Holocaust in the age of decolonization, Stanford, Stanford university press, 2009.
} 
geographies of memory where monuments competed for attention in terms of urban space, positioning, and reception', ${ }^{8}$ esplicitando che le memorie divise riguardano non solo le commemorazioni e altri segni visibili di pratiche memoriali, ma anche chi o ciò che si oppone a tali attività. Quest'ultimo gruppo, percepito in un primo momento negativamente come un insieme di autori di eventuali danneggiamenti, diventa involontariamente il motore di tentativi di recupero. In questo senso i murales propongono un discorso commemorativo contro l'oblio, ${ }^{9}$ in cui si intrecciano microstorie, quali testimonianze di (parenti di) vittime che spesso circolano tramite $i$ media entrando nella memoria socio-culturale e formando memorie collettive, ${ }^{10}$ le quali a loro volta possono (ri)generare discorsi contro-egemonici dal basso, come già sostenuto da Gramsci. ${ }^{11}$

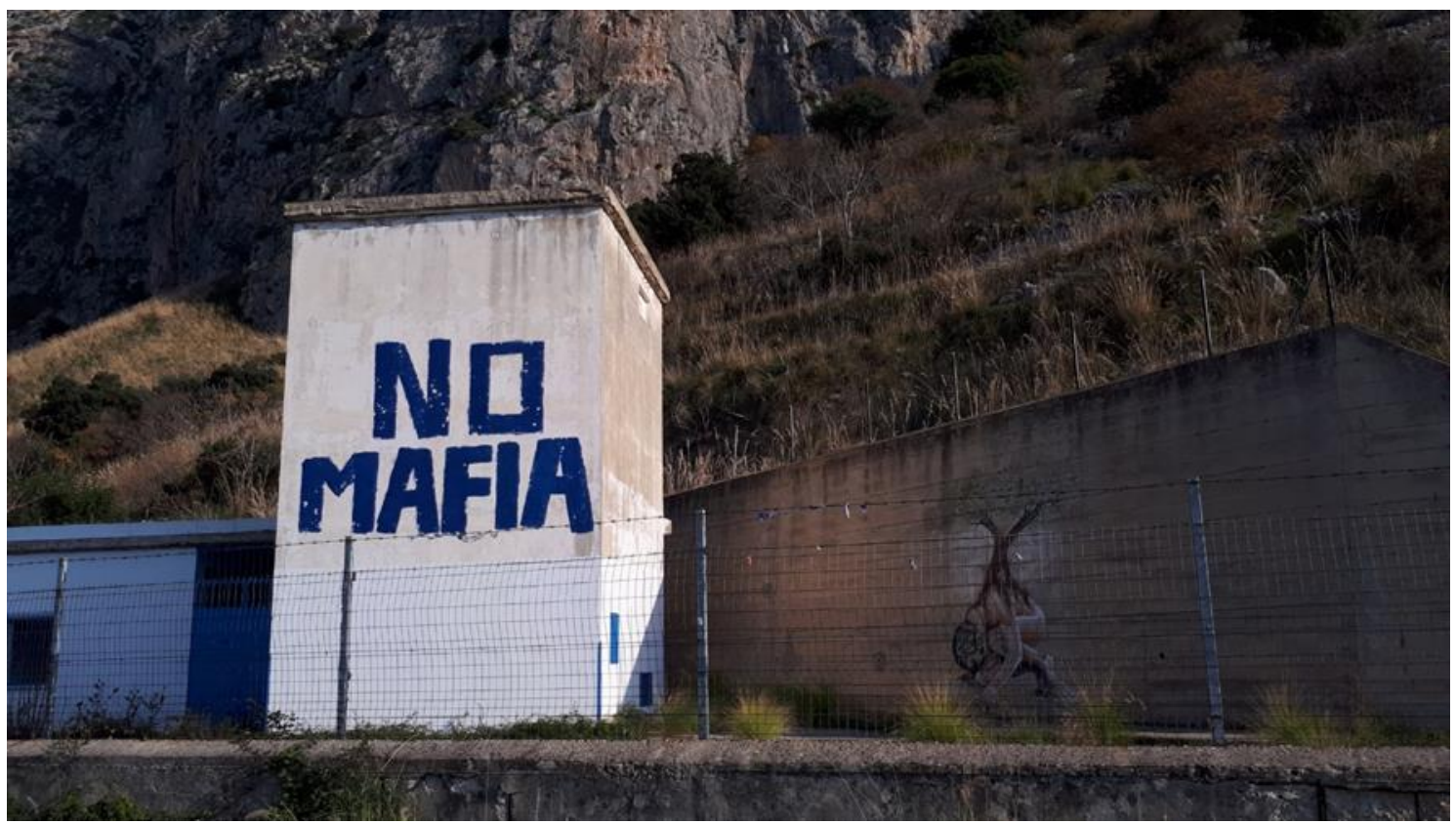

Foto 1: Il murales ‘no mafia’ a Capaci @ Inge Lanslots-Paul Sambre-Eliana Maestri-Sidney Cardella

\section{Protesta spontanea}

Sia il murales di Capaci che quello della Garbatella possono essere letti come facenti parte integrante della street art anche se il loro ripristinamento, ripetitivo o meno, sembra contraddire la natura intrinsecamente effimera delle opere murarie. ${ }^{12}$ Laddove le opere murarie sono solite sparire, si cede il posto ad altre opere mettendo in

\footnotetext{
8 J. Foot, Italy's Divided Memory, New York, Palgrave Macmillan, 2009, pp. 4-5.

${ }^{9}$ Ivi, p. 6.

${ }^{10}$ A. Erll, Memory in Culture, New York, Palgrave Macmillan, 2011, pp. 97, 107-108 e 128.

11 P. Sambre, 'Multimodal and Socio-cognitive Explorations of Counterhegemonic Discourse: Anti-mafia Documentaries and Field Work', presentato al workshop intitolato 'Dottorato di Ricerca di Studi Linguistici, Letterari e Interculturali in ambito europeo ed extra-europeo', Polo di Mediazione Interculturale e Comunicazione, Università Statale di Milano, 28 settembre 2019.

12 Durante la ricerca sul campo del gruppo di ricerca, a cui durante il soggiorno a Palermo si è aggiunto Sidney Cardella (KU Leuven), gli esperti e le guide hanno insistito molto sull'importanza della documentazione fotografica che potrebbe fungere da archivio indispensabile per i (non) addetti ai lavori. Tramite tale documentazione, la presenza o l'esistenza dell'opera muraria in sé effimera viene prolungata e l'opera stessa può continuare a circolare in rete sottraendosi a forme di censura o di ripristinamento. Per la natura effimera delle opere murarie si consulti: J. Ferrell, 'Foreword. Graffiti, Street Art and the Politics of Complexity', Routledge Handbook of Graffiti and Street Art, London, Routledge, 2016, pp. xxx-xxxviii, in particolare p. xxxiv.
} 
evidenza l'eventuale degrado del quartiere in cui sono nate e, al contempo, la volontà di riscrittura continua. $\dot{E}$ in questo quadro che si inseriscono le opere prese in considerazione nel presente contributo, opere che perpetuano il processo creativo valorizzando maggiormente il carattere dinamico dei murales in quanto pratica artistica.

In questo rispetto, i due murales condividono una storia simile. Grazie alla documentazione fotografica, si possono registrare vari tipi di cancellazioni dovute sia all'esposizione agli elementi naturali, che a interventi su opere esistenti o, più semplicemente, causate da una sovrapposizione parziale di un'opera nuova o da una rimozione per mano di un intervento istituzionale, come quello apportato alla frase 'Sei la mia vita' apparso sotto la scritta 'no mafia'. Oltre a ciò, i murales hanno in comune una dimensione processuale, fortemente legata a quella commemorativa, anche se quest'ultima non è automatica nella street art - la regola di base della street art è quella di parlare liberamente alla e della città. ${ }^{13}$

Nell'ambito dei memory studies, la dimensione processuale legata alle opere pubbliche, in cui il ricordo e la preservazione vanno di pari passo, combacia con la formazione di un palinsesto che rivela una tensione nello spazio pubblico. ${ }^{14} \mathrm{Nel}$ caso di 'no mafia', tale tensione va interpretata come atto di rivendicazione spontanea: con la scritta 'no mafia' a caratteri cubitali il fotografo Antonio Vassallo e altri abitanti di Capaci fin da subito hanno voluto rioccupare lo spazio che i mafiosi, sotto la guida di Brusca, avevano usato per innescare l'esplosivo che distrusse il tratto dell'autostrada A29, alle 17.58 del 23 maggio 1992, al momento del passaggio delle cinque macchine della scorta del giudice Falcone. Come riferito da Vassallo durante l'intervista, tenutasi il 12 gennaio 2020, al momento della strage, che fu una delle risposte di Cosa nostra alla sentenza del maxiprocesso del 30 gennaio dello stesso 1992, il fotografo si trovava per puro caso nelle vicinanze e fece presto ad arrivare in loco dopo che una carica ad altissimo potenziale, posta in un sottopassaggio, fece sollevare il manto dell'autostrada, creando una voragine di quasi ventri metri. Vassallo stette vicino a Falcone, che quel giorno stava alla guida della macchina, una pratica approvata negli anni ottanta, finché nell'immediato caos le forze dell'ordine lo cacciassero via, perché nella confusione di quel momento lo avevano scambiato per un eventuale complice. In un breve lasso di tempo il fotografo riuscì a registrare i fatti le sue foto furono confiscate - e la sua indignazione nata in quei momenti tragici si converti ben presto in un impegno sociale continuo. Grazie alla collaborazione del centro sociale di Capaci e dei suoi giovani, che poi fondarono Addiopizzo e/o Addiopizzo Travel, ${ }^{15}$ egli coprì il muro del casolare Amap con la scritta 'no mafia', lanciando un appello ben visibile dall'autostrada. Anche se il casolare si trova su un terreno privato, la scritta è liberamente visibile al pari di altre opere murarie, chiaro indizio dell'immediatezza tipica della street art. In questa parte della collina si sono poi organizzate - e si organizzano tuttora - non solo manifestazioni commemorative, ma anche numerose visite che coinvolgono sia le gite scolastiche che le escursioni di chi si presta all'impegno antimafia. ${ }^{16}$ Alla volontà di commemorazione si associa quindi un lavoro di sensibilizzazione che a sua volta si iscrive in un programma più ampio di

\footnotetext{
${ }^{13}$ M. Filippi, M. Monolini \& L. Tuttolomondo, Street art in Sicilia. Guida ai luoghi e alle opere, Palermo, Dario Flaccovio, 2017, pp. 24 e 7.

${ }^{14}$ Foot, Italy's Divided Memory, cit., p. 4.

15 Cfr. i rispettivi siti, seppur connessi, delle due associazioni: https://www.addiopizzo.org/ e https: / /www.addiopizzotravel.it/. La scelta del Comitato Addiopizzo Travel è stata quella di stabilire la sede a Isola delle Femmine, sul cui territorio comunale avvenne la strage del 1992, mentre la sede di Addiopizzo ha sede a Palermo, in un appartamento confiscato alla mafia.

${ }^{16}$ Le commemorazioni vengono annunciate e celebrate sui siti delle associazioni appena menzionati, ma anche sui siti di giornali regionali. A questo proposito va sottolineato quanto Facebook e altri social media siano importanti nel comunicare le notizie e nel commentare gli eventi.
} 
un consumo critico e di un'etica non solo antimafia ma anche antimafiogena. ${ }^{17} \mathrm{La}$ street art svolge chiaramente un ruolo fondamentale nell'attivismo sociale, il che porta Vlad Glăveanu ad affermare che: 'Creative activism is thus not a sub-form of social activism but a quality of activist or socially engaged action."18 Nell'interpretazione di Lisa Bogerts il ruolo della street art nell'attivismo viene riformulato in funzione degli attori sociali stessi: 'people can challenge or resist dominant ideology with the help of the visual and, in turn, foster awareness'. ${ }^{19}$

Proprio nell'ambito dell'attivismo, Addiopizzo e Addiopizzo Travel hanno usufruito delle possibilità offerte dalla street art per poter contribuire a formare una maggiore consapevolezza della lotta alla mafia. Ambedue le associazioni sono state tra le prime a discutere in modo aperto e sistematico l'impatto del racket sulla Sicilia e la consecutiva percezione dei meccanismi di Cosa nostra facilitando cosi, da una parte, denunce individuali e collettive e, dall'altra, una solidarietà tra commercianti. Instaurando un dialogo costruttivo con altri attori sociali e movimenti, quali Umberto Santino, Nando Dalla Chiesa e l'Osservatorio sulla Criminalità Organizzata, il Centro Siciliano di Documentazione Giuseppe Impastato, il No Mafia Memorial e Libera. Associazioni, nomi e numeri contro le mafie, ${ }^{20}$ esse s'impegnano a promuovere la cultura della legalità che si oppone apertamente alla cultura mafiosa. ${ }^{21} \mathrm{Nel}$ giro di quindici anni tali movimenti dal basso hanno progressivamente acquistato il consenso del pubblico, anche se sono ben consci della lunga strada che rimane da percorrere, perché l'organizzazione criminale è tuttora presente e attiva in Sicilia e oltre, seppure in modo meno vistoso. In più occasioni gli attivisti, le guide e gli esperti, intervistati durante la nostra ricerca sul campo a Palermo nel gennaio del 2020, hanno insistito sulla trasformazione di Cosa nostra: dopo la violenza estrema degli anni ottanta e novanta, l'organizzazione criminale siciliana si è sentita costretta ad adottare un riposizionamento nei confronti degli organi istituzionali nonché di altre organizzazioni criminali pur non interrompendo le proprie attività e ritirandosi però in un'invisibilità

\footnotetext{
${ }^{17}$ Concetto proposto da Umberto Santino durante il suo laboratorio 'Sulla borghesia mafiosa e sul concetto di mafiogeno', tenutosi il 16 Gennaio 2020 al No Mafia Memorial di Palermo. Con 'mafiogeno' Santino intende identificare atteggiamenti e pratiche nella vita quotidiana che richiamano quelli prettamente mafiosi - basti pensare al sistema di clientelismo, di scambi e di favoritismi.

18 V.P. Glăveanu, 'Art and Social Change: The Role of Creativity and Wonder', in: S.H. Awad \& B. Wagoner (a cura di), Street Art of Resistance, Cham, Springer International Publishing-Imprint Palgrave Macmillan, 2017, p. 20.

19 L. Bogerts, 'Mind the Trap: Street Art, Visual Literacy, and Visual Resistance', in: Street Art \& Urban Creativity Scientific Journal, 3, 2(2017), p. 10.

20 I siti rispettivi degli attori sociali appena menzionati sono: https://www.nandodallachiesa.it/, https://www.centroimpastato.com/, http://www.nomafiamemorial.org, https://www.libera.it (18 agosto 2020). Va esplicitato che gli attori sociali, le varie associazioni, anche attivi su altri social media, spesso collaborano per lanciare appelli e per organizzare eventi o altre iniziative.

Nell'ambito del presente contributo va messo in evidenza che i ricercatori, gli attivisti e le associazioni lottano contro la criminalità organizzata impegnandosi a mantenere viva la memoria e dedicandosi allo stesso tempo alle proprie attività di ricerca. Libera Memoria, per esempio, aspira sia a 'mantenere vivo il ricordo delle vittime innocenti delle mafie sia di camminare al fianco dei loro familiari, organizzando momenti di confronto e formazione' (https://www.libera.it/schede-2-memoria_e_impegno, 18 agosto 2020).

Va aggiunto che il banner del No Mafia Memorial, che ha sede in un bene confiscato alla mafia nel centro di Palermo, riecheggia la calligrafia della scritta del murales.

${ }^{21}$ Per ulteriori informazioni sul rapporto tra la cultura della legalità

- e la lotta antimafia, agevolato dai nuovi media, si consulti: R. Pickering-lazzi, The Italian Antimafia, New Media, and the Culture of Legality, Toronto, University of Toronto, 2017;

- e la lotta antimafia, quale l'antipizzo, si consulti: E. Savona, 'Italian Mafias' Asymmetries', in: Traditional Organized Crime in the Modern World, Boston MA, Springer, 2012, pp. 3-25;

- e le vittime delle organizzazioni criminali, si consulti J. van Dijk,'Transnational Organized Crime, Civil Society and Victim Empowerment', in: The New Faces of Victimhood, Dordrecht, Springer Netherlands, 2010, pp. 99-126.
} 
apparente. Tale contesto rivela che mentre le indagini sulle ramificazioni transnazionali di Cosa nostra sono sempre in corso, le attività antimafia, di cui il murales 'no mafia' è un simbolo chiave, continuano a intensificarsi. Come ha testimoniato in più occasioni Dario Riccobono, co-fondatore e portavoce principale di Addiopizzo Travel, il murales dà voce e forma dal basso alla società civile: 'Quel luogo per iniziativa spontanea di tanti comuni cittadini, è diventato simbolo di resistenza alla mafia e di riscatto, cartolina di una Sicilia che resiste'. ${ }^{22}$ Data la collocazione il murales viene inoltre subito notato dai turisti stranieri o meno che si recano in varie parti della Sicilia dall'aeroporto internazionale di Palermo-Punta Raisi, ribattezzato 'Falcone e Borsellino'. Insieme al monumento formato da due obelischi ai due lati dell'autostrada Palermo-Trapani e al 'Giardino della memoria', rispettivamente eretto nel 2004 e rivalorizzato nel 2015, la scritta in alto sulla collina ormai andrebbe vista come una prima porta d'accesso alla città di Palermo. ${ }^{23}$ Nel caso di Capaci, il summenzionato monumento alla memoria dei caduti della mafia assume dimensioni sempre maggiori, anche a livello simbolico - lo illustra il nuovo nome del parco vicino al luogo dell'attentato 'IV Savona 15' che rimanda a quello dell'auto della scorta, e viene appoggiato dalle autorità locali e regionali, tra cui il primo cittadino di Palermo Leoluca Orlando.

Nonostante il consenso, sia della società civile che dell'amministrazione, e l'interesse di cittadini italiani e altri, europei o meno - ne sono testimonianza le molte visite guidate e altre attività - gli intervistati sono ben consci del fatto che il murales non sembri accogliere chi non abbraccia la causa né l'impegno antimafia. A Capaci si soffermano solo gli abitanti e chi vuole visitare il monumento, e il comune si oppone a rappresentanti politico-istituzionali contrari alle idee appoggiate dai movimenti dal basso (i grassroots). Così, alla commemorazione antimafia annuale del 2019, la presenza dell'allora ministro dell'Interno Matteo Salvini non è stata gradita, proprio perché l'attivismo antimafia si sta inserendo sempre di più in un dibattito più ampio: alla lotta antimafia, nella visione di Addiopizzo e Addiopizzo Travel, si ricollegano volutamente le questioni di occupazione, di migrazione, di ambiente, di formazione e di informazione, argomenti su cui Salvini esprime visioni provocatorie diffondendo spesso notizie false e non comprovate. A Capaci, il murales rimane quindi una memoria divisa: un'opera circondata da una certa ambiguità e da una forza centrifuga che porta in superficie la polarizzazione ideologica tra la sinistra e la destra italiana.

\section{'Memoryscapes' e geografie urbane}

La tensione tangibile nello spazio pubblico illustra chiaramente quanto i 'memoryscapes' e le geografie urbane che ospitano murales generino un discorso commemorativo contro l'oblio. ${ }^{24}$ Nelle microstorie che nascono in questo con-testo si inseriscono le testimonianze di (parenti di) vittime, le memorie collettive, menzionate nella parte introduttiva, che contribuiscono a costruire un nuovo discorso contro-

\footnotetext{
22 D. Riccobono in 'No Mafia: coloriamo i nostri sogni', in: ANTIMAFIADuemila. Informazioni su Cosa Nostra e organizzazioni criminali connesse, 16 maggio 2017, http://www.antimafiaduemila.com/home/ rassegna-stampa-sp-2087084558/cronache-italia/65498-no-mafia-coloriamo-i-nostri-sogni-2.html (18 agosto 2020).

Cfr. anche: 'L'origine della scritta "NO MAFIA", un urlo diventato simbolo di resistenza', in: ANTIMAFIADuemila, 24 maggio 2018, http://www.antimafiaduemila.com/home/rassegna-stampa-sp2087084558/114-mafia-flash/70452-l-origine-della-scritta-no-mafia-un-urlo-diventato-simbolo-diresistenza.html (18 agosto 2020$)$.

${ }^{23}$ Tra i vari commenti pubblicati all'epoca sul nuovo monumento e sul parco, si legga, per esempio, 'Un parco in memoria di Falcone lungo l'A29, arriva il “IV Savona 15"', in: PALERMOTODAY, 15 maggio 2015, https://www.palermotoday.it/cronaca/autostrada-capaci-parco-falcone.html (24 settembre 2020).

${ }^{24}$ Foot, Italy's divided memory, cit., p. 6.
} 
egemonico dal basso. ${ }^{25}$ Così, in occasione della manifestazione 'No Mafia, coloriamo i nostri sogni: 1992-2017', per la commemorazione del venticinquesimo anniversario della strage, Brizio Montinaro, fratello di una delle vittime, ha sostenuto che il luogo non richiede 'discorsi di parata', ma l'impegno degli individui a livello glocale. ${ }^{26}$

Rispetto alla tensione che caratterizza il murales di Capaci, quella attivata dal murales della Garbatella sembra più acuta e allo stesso tempo più puntuale, culminando in un momento anti climax nell'ottobre del 2016: momento in cui, come riferito prima, il Municipio VIII decise di togliere il murales dedicato alla memoria di Carlo Giuliani. Fu allora che scomparvero alcune immagini iconiche quali quella in cui vediamo Carlo da dietro mentre tiene in mano l'ormai noto estintore. Dopo le prime polemiche scatenate sui social media, la ripulitura in Via Cialdi fu coperta da nuovi murales. Il murales alla memoria di Giordani fu tuttavia ripristinato per mano degli attivisti del centro sociale di Via Passino della Garbatella, La Strada, fondato nel 1994, a soli cinque giorni dalla cancellazione della prima opera a lui dedicata. Con tale ripristino gli attivisti del centro sociale vollero ricaricare il quartiere della Garbatella, non solo noto in quanto espressione del piano edilizio-urbanistico degli anni venti ma anche in quanto zona rossa e operaia, del valore simbolico che lo aveva caratterizzato prima della cancellazione del murales alla memoria di Giordani. In questo contesto, il murales diventa simbolo di resistenza al potere istituzionale e amministrativo e, al contempo, di memoria collettiva. Non viene strumentalizzato da operazioni di turistificazione o di 'city branding', né viene percepito come una muralizzazione gratuita, senza un vero messaggio o un destinatario. ${ }^{27}$ In un'intervista su espressioni di disordine o disagio sociale, espressioni sintomo di una ricerca di affermazione individuale, la filosofa-sociologa Tamar Pitch, che sviluppa un interesse particolare in questioni giuridiche, sottolinea quanto i cittadini sentano il bisogno di nobilitarsi protestando tramite opere murarie lette talvolta dalle autorità come manifestazioni di degrado o di mancanza di decoro. ${ }^{28}$ Di recente Ann Rigney ha evidenziato quanto venga sottovalutato, nell'ambito dei memory studies, il nesso tra la trasmissione di positività e l'impegno nei confronti di valori sociali. ${ }^{29}$ Molte opere murarie, infatti, vengono interpretate troppo facilmente all'insegna del 'traumatic paradigm that both responds to, and feeds into, the predominance of mourning and memorialisation in contemporary cultures of memory', mentre in realtà costituiscono forme culturali che generano pratiche positive tramite cui si effettua la trasmissione dell'impegno civico. ${ }^{30}$

Come diventerà chiaro, la tensione tangibile scaturita dalla percezione della street art generata a seguito dello svolgimento del G8 ricalca quella che circonda la memoria di Carlo Giuliani stesso. ${ }^{31}$ Subito dopo la morte del giovane, i media fecero

${ }^{25}$ Erll, Memory in Culture, cit., pp. 97, 107-108 e 128.

26 'Capaci, ridipinta la scritta "No mafia”: il primo a colorarla è il fratello di Montinaro', in: PALERMOTODAY, 22 maggio 2017, https: / /www.palermotoday.it/cronaca/strage-capaci-ridipinta-scrittano-mafia.html (18 agosto 2020).

${ }^{27}$ Cfr. S. Andron, 'Selling Streetness as Experience: The Role of Street Art Tours in Branding the Creative City', in: The Sociological Review, 66, 5 (2018), pp. 1036-1057.

28 C. Antonucci, 'Decoro oggi: disordine o disagio sociale? Intervista a Tamar Pitch', in: CORE. Circuiti Organizzati Resistenze Editoriali, 02 febbraio 2015, http://www.csoalastrada.net/coreonline/resistenze-2/decoro/ (18 aprile 2020).

${ }^{29}$ A. Rigney, 'Remembering Hope: Transnational activism beyond the traumatic', in: Memory Studies, 11, 3 (2018), p. 368. Il contributo di Rigney mette a fuoco la lacuna della ricerca a questo proposito.

30 Rigney, 'Remembering Hope: Transnational activism beyond the traumatic', cit., pp. 369 e 372.

${ }^{31}$ Cfr. M. Jansen \& I. Lanslots, 'Dalla parte del noir: l'oscura immensità del G8 a Genova 2001', in: M. Jansen, I. Lanslots \& D. Vermandere (a cura di), Noir de noir, Brussels, Peter Lang, 2010, pp. 73-88; Id., 'Piazza Alimonda's open wound: The memory site of Genoa 2001', in: S. Storchi (a cura di), Beyond the Piazza. Public and Private Spaces in Modern Italian Culture, Brussels, Peter Lang, 2013, pp. 215-227. I. Lanslots, 'La trasmissione della memoria del G8 2001: due percorsi grafici dell'irruzione nella Diaz-PertiniPascoli', in: Narrativa, 35 (2014), pp. 207-219; Id., 'Gli ingranaggi della memoria del G8 2001 nella fumettistica italiana: Carlo Giuliano, né eroe né martire’, in: Forum Italicum, 2017, pp. 112-132. 
nascere una polemica attorno alla sua figura: invece di essere ritratto in qualità di attivista e vittima di violenza, Giuliani fu rappresentato dai media come un drogato, un punkkabestia o addirittura un esponente dei Black Bloc(k). ${ }^{32}$ Varie istituzioni statali sembrarono addirittura cancellare il ricordo di Giuliano: le indagini sulla sua morte furono sbrigative; i vari processi in cerca di giustizia e di verità per Giuliani e le altre vittime di violenza subita durante lo stesso vertice, coordinati dal Genoa Legal Forum e raccolti sul sito dell'archivio Antimafia, ${ }^{33}$ portarono a proscioglimenti o a sentenze poi revocate. Anche la città di Genova emanò vari divieti per fermare l'organizzazione di iniziative commemorative nella piazza dove avvennero i fatti fatali. Si registrarono tentativi di far rimuovere il cippo dedicato a Giuliani in piazza Alimonda ${ }^{34}$ e l'altare laico, fino alla posa ufficiale di una lapide nel 2005. Il piano di ribattezzare la piazza in nome di Carlo Giuliani fu ostacolato fino al decimo anniversario della morte del giovane. Dal 2011 in poi, l'anno in cui fu posta la nuova targa, il nuovo nome della piazza divenne l'espressione

di fenomeni di ritorno a un'odonomastica dal basso, spontaneamente attribuita come quella di età preunitaria, ma portatrice della stessa funzione ideologica, politica e memoriale di cui oramai i procedimenti di intitolazione sono inevitabili portatori, siano espressione del potere costituito o della libera iniziativa popolare. ${ }^{35}$

Il ripristinamento del murales della Garbatella ravviva la memoria traumatica di una delle stragi più recenti della storia italiana, poco nota al di fuori dall'Italia. Per chi invece ha familiarità con lo svolgimento del G8 del 2001, la visualizzazione del giovane all'entrata del quartiere permette di re-interpretare il trauma alla luce degli eventi trascorsi a seguito del summit politico, inclusi gli episodi di violenza da parte dei manifestanti, in particolare dei Black Bloc(k), e delle forze dell'ordine nonché dei relativi discorsi generati a seguito dell'incomprensione verso quanto accaduto:

Not the content of the event, but the incomprehensibility of the scenario in which the event occurred - inseparable from the affective memories that it elicits - is the crucial instance that provokes the traumatic return. While the emergence of the traumatic scenario defies linguistic articulation, its manifestation triggers the bodily memory of the sensorial and affective components associated with that experience. ${ }^{36}$

Alla luce di questa lettura, la scritta a destra sul primo muro della scalinata 'A.C.A.B.' ('All Cop(per)s Are Bastards'), assume un ulteriore valore simbolico completando la richiesta di libertà da parte degli abitanti del quartiere situata a sinistra del murales

\footnotetext{
32 Sono accettate le due forme del termine, coniato dalla polizia tedesca negli anni ottanta per individuare manifestanti della sinistra extraparlamentare, vestiti di nero, che non esitano a usare la violenza. Dagli anni novanta il termine viene usato per indicare gli individui, sempre vestiti di nero, che fanno incursioni in modo violento in cortei e manifestazioni. La prima comparsa in Italia risale appunto al vertice del G8 tenutosi a Genova.

33 Cfr. https://www.archivioantimafia.org/www.processig8.org/index.html, il link che raccoglie le informazioni sullo svolgimento delle indagini e dei processi (le sentenze e i vari proscioglimenti), sul ruolo dei responsabili, sugli abusi delle forze dell'ordine; cfr. l'obiettivo del Comitato Verità e Giustizia per Genova: 'Il Comitato organizza iniziative volte alla tutela delle vittime della repressione delle forze dell'ordine nell'esercizio della manifestazione del pensiero, anche con l'utilizzo degli strumenti di azione regolati e previsti dal diritto internazionale con particolare riferimento a quelli creati nell'ambito dell'Unione Europea' (veritagiustizia.it, 18 aprile 2020).

${ }^{34}$ Cfr. Comitato Piazza Carlo Giuliani (Piazzacarlogiuliani.it, 18 aprile 2020).

${ }^{35}$ M. Pentucci, 'La storia per le strade', in: C. Gaetani (a cura di), L'identità nazionale. Storie, film e miti per raccontare l'Italia, Ancona, Affinità Elettive, 2011, p. 178, https://www.researchgate.net/ publication/280254355_La_storia_per_le_strade (18 aprile 2020).

${ }^{36}$ P. Lešnik, 'Pavese, Antonioni, and the Specters of a Silenced Past: Adaptation and the Transmission of Historical Trauma', in: Adaptation, 12, 1 (2019), pp. 1-11.
} 
principale (Foto 2-3-4). Nel murales che, come ha illustrato Fabio Barilari durante la visita guidata, è frutto della guerriglia painting tipica e continua del quartiere ${ }^{37} \mathrm{si}$ nota anche l'estintore dipinto su un armadio ripartilinea che, contrariamente a quello tramandato dall'iconografia della strage, è stato separato dall'immagine di Carlo stesso. Il trittico all'ingresso della Garbatella è un'altra denuncia della violenza compiuta dalle forze dell'ordine durante tutto lo svolgimento del G8 2001, dalla morte di Carlo Giuliani attraverso l'irruzione notturna nella scuola Diaz-Pertini-Pascoli ai pestaggi alla caserma di Bolzaneto: tutte immagini di violenza il cui peso è ormai inciso nella memoria collettiva italiana. Il dialogo che si stabilisce tra la scritta di natura anarchica, la dichiarazione di libertà e la grande opera muraria centrale sottolinea la ferita aperta lasciata dai fatti di Genova, una ferita che tende comunque a 'ridisegnare' l'iconografia tramandata dai fatti.

Nel caso del murales dedicato alla memoria di Giuliani, il tentativo di trasformare l'iconografia e l'immaginario collettivo legato al G8 2001 si manifesta a un altro ritmo rispetto a quello realizzato in Sicilia con 'no mafia'. Il contesto di violenza che circonda la figura del giovane genovese è indubbiamente più ambiguo. A Capaci si è inclini ad affermare che la scritta 'no mafia' sembri instaurare un dialogo esplicitamente costruttivo tramandando un messaggio di rigenerazione di Palermo e della Sicilia per estensione, dei cittadini individuali nonché delle comunità mosse dalle azioni di individui. Questo messaggio si visualizza ulteriormente tramite l'opera muraria vicino alla scritta in azzurro che rappresenta un ragazzo nudo in una posizione rannicchiata, quasi fetale (Foto 1, a destra), sul cui dorso cresce un albero. L'immagine del secondo murales rappresenta la resilienza nonché la vulnerabilità dei siciliani nella lotta antimafia ma costituisce al contempo un omaggio originale alle vittime della mafia. L'albero ivi dipinto richiama l'Albero Falcone a Palermo in via Notarbartolo 23, piantato davanti alla casa del giudice assassinato, che, dal momento della strage, si è trasformato in un luogo di memoria dove vengono lasciati messaggi, fotografie e oggetti. L'Albero Falcone, a sua volta, ha portato all'iniziativa degli 'Alberi Amici [...] piantati da chiunque desidera vedere germogliare un altro Albero Falcone in un giardino pubblico della sua città, nel cortile della sua scuola o in qualsiasi spazio che lo possa accogliere'. ${ }^{38}$ L'albero raffigurato nel murales a destra di 'no mafia' è emblematico delle attività della resistenza esercitata nell'ambito dell'attivismo antimafia, ovvero nell'ambito della collaborazione 'orizzontale' tra vari attori pubblici, privati e no profit. ${ }^{39}$ In questo modo, le opere murarie, insieme ad altri luoghi

\footnotetext{
37 La guerriglia painting si estende a tutto il quartiere popolare dell'Ostiense, con scritte e immagini di proteste nonché esempi di stickering su pali della luce o sulla segnaletica stradale, esempi presenti nella foto 4. A questo scopo vanno letti: M. Tissino Di Giulio, 'Nuove forme di resistenza attraverso il Murales: il caso di una periferia romana', in: H-ermes. Journal of Communication, 9 (2017), pp. 229-240; per la storia del quartiere si consulti invece A. De Michelis, "“Civis romanus sum": The Self-Conscious "Romanità" of the Garbatella', in: Annali d'Italianistica, 28 (2010), pp. 153-178.

La guerriglia painting s'iscrive nella lunga tradizione di protesta del quartiere, cfr. G. Gambetta \& G. Furini (a cura di), Abitare a Roma in periferia / Living in Rome in the Suburbs. Fotografie di Rodrigo Pais / Photographs by Rodrigo Pais, Roma, Gangemi, 2016; G. Rivolta, Dalla Villetta ai Gazometri. Partiti politici e lotte popolari nel dopoguerra tra Garbatella e Ostiense, Roma, lacobelli, 2012; G. Rivolta (a cura di), Garbatella 100. Il racconto di un secolo, Roma, lacobelli, 2019.

38 Per ulteriori informazioni, foto e contestualizzazioni si consulti: 'Gli Alberi Amici', http://www.fondazionefalcone.it/alberi-amici/ (24 settembre 2020).

L'iniziativa di piantare nuovi 'Alberi Falcone' in spazi pubblici quadra nella missione pedagogico-educativa della Fondazione Falcone, fondata nel 1992 e rivolta in primo luogo ai ragazzi in età scolare. Nel giro degli anni le attività della fondazione si sono però diversificate, motivo per cui il sito si è trasformato in un portale vero e proprio, con fonti, riferimenti e corsi che si rivolgono ad attivisti e studiosi.

39 Per le origini e una definizione più elaborata del concetto, si consultino: D. Mackinnon \& K. Driscoll Derickson, 'From Resilience to Resourcefulness: A Critique of Resilience Policy and Activism', in: Progress in Human Geography, 37, 2(2012), pp. 253-270; J. Schneider \& P. Schneider, 'Sicily: Reflections on Forty Years of Change', in: Journal of Modern Italian Studies, 11, 1 (2006), pp.61-83.
} 
di memoria dinamici, creano realtà, mondi e network cambiando il presente e disegnando il futuro. ${ }^{40}$ In questo contesto le strategie che hanno ispirato tali opere murarie finiscono a loro volta per 'inspire groups of activists in different contexts to reclaim their own environments through the coupling of self-organization of social reproduction and political pressure via social movements'. ${ }^{41}$
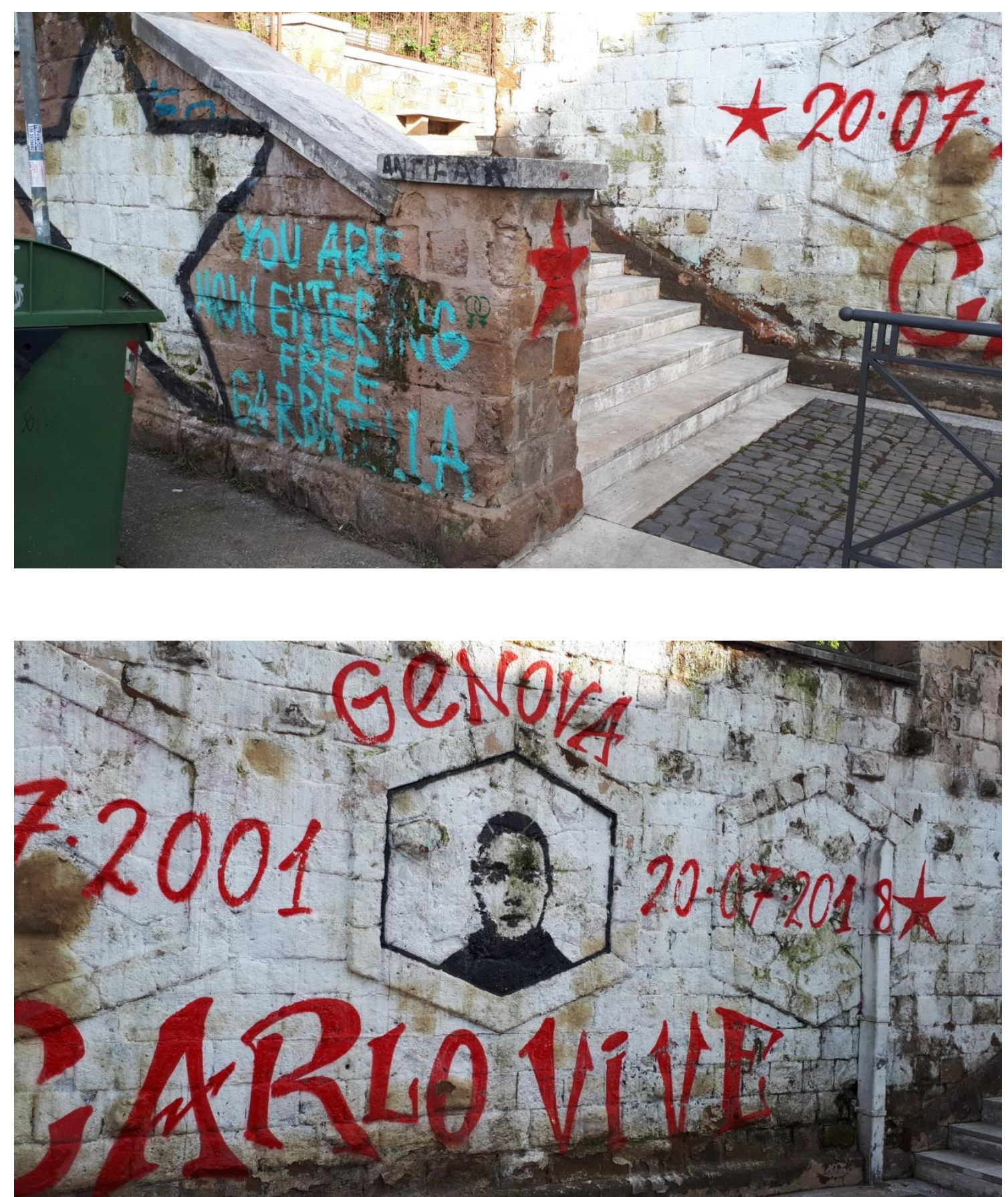

40 Glăveanu, ‘Art and Social Change: The Role of Creativity and Wonder', cit., pp.33-34.

${ }^{41}$ M. Caggiano \& S.P. De Rosa, 'Social Economy as Antidote to Criminal Economy. How Social Cooperation is Reclaiming Commons in the Context of Campania's Environmental Conflicts', in: The Open Journal of Sociopolitical Studies, 8, 2(2015), p. 554, http://siba-ese.unisalento.it/index.php/paco (20 agosto 2020). 


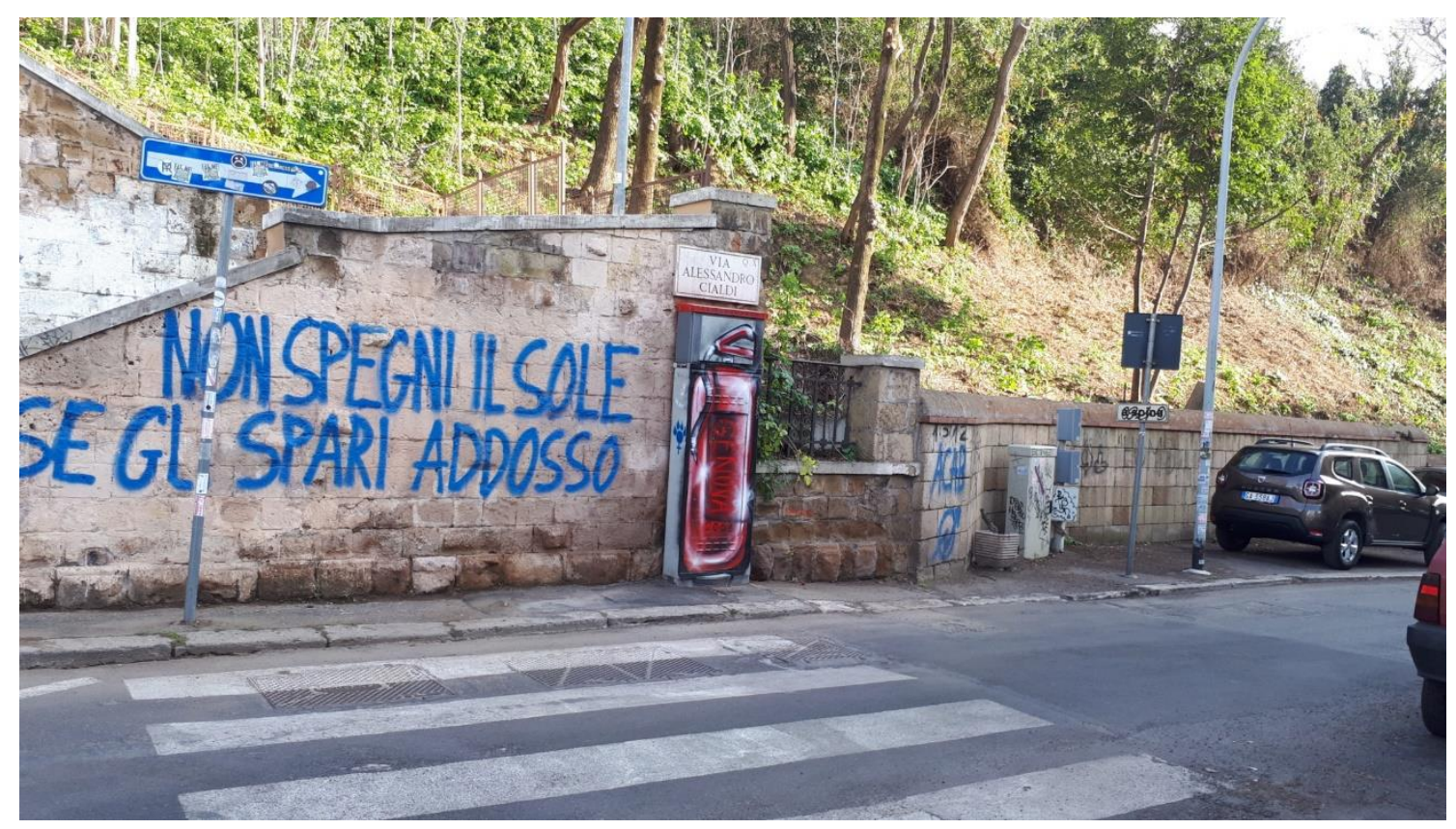

Foto 2-3-4: Il murales dedicato a Carlo Giuliani all'ingresso della Garbatella @ Inge Lanslots

\section{'Being-in the street'"42}

Parafrasando le parole di Dario Riccobono di Addiopizzo, simili a quelle di altri attivisti e di esperti, le opere murarie prese in considerazione non sono lavori commissionati che aspirano a una riqualificazione dei quartieri in questione. Al contrario, sono la testimonianza della presenza dell'attivismo civico nel tessuto urbano, sia esso periferico o meno. ${ }^{43}$ Nate in modo spontaneo, e originariamente in modo anonimo, tali opere riflettono 'azioni community specific' ${ }^{44}$ formando luoghi di resistenza, facendo appello alla coscienza pubblica e richiedendo un impegno quotidiano. Proprio per questo, gli attivisti insistono sul fatto che il meravigliarsi, l'interrogarsi e l'indignarsi non basti più: ${ }^{45}$

Nel conflitto già in corso, i muri delle città sono sia sfondo che parti in causa. Come da sempre è nella loro vocazione[:] veicolano rabbia e malcontento, tifo sportivo e impegno politico, messaggi d'amore e di poesia. C'è chi continuerà a scrivere e chi a cancellare $\left[\ldots . .{ }^{46}\right.$

Tocca poi alla critica continuare la ricerca sui muri relativi alle stragi e sul dialogo su di essi, dialogo che si espande ad altre città in cui spuntano opere simili. In questa ricerca vanno inclusi lo studio degli aspetti discorsivi relativi alla positività, come, per esempio, 'No Mafia, coloriamo i nostri sogni', l'analisi della calligrafia dei caratteri scritti cubitali, e dell'uso delle minuscole che vogliano ridimensionare la mafia, nonché l'indagine su come $i$ luoghi fisici si perpetuino online tramite foto e commenti postati su vari social media.

\footnotetext{
${ }^{42}$ Cfr. Kenaan, 'Streetography: on Visual Resistance', cit., p.152.

43 Salvi, 'Muri e memoria: la guerra del decoro che ingrigisce le città', cit.

${ }^{44}$ Filippi, Monolini \& Tuttolomondo, Street art in Sicilia. Guida ai luoghi e alle opere, cit., p. 56.

45 Glăveanu, 'Art and Social Change: The Role of Creativity and Wonder', cit., p. 34.

${ }^{46}$ Salvi, 'Muri e memoria: la guerra del decoro che ingrigisce le città', cit., grassetto originale.
} 
Parole chiave

attivismo, antimafia, Carlo Giuliani (G8 2001), street art, memory studies

Inge Lanslots è professore associato di traduzione e di cultura italiana presso l'Università di Lovanio (KU Leuven) ed è membro del gruppo di ricerca VICT (Traduzione e transfer interculturale). Specializzatasi nella narrativa italiana contemporanea, ha scritto vari contributi su più autori (Baricco, Benni, Ortese, Pressburger, Tabucchi), ma anche sul giallo/noir, sui (cant)autori, sulla fantascienza, sui fumetti e sul romanzo grafico, nonché sull'intermedialità, si dedica ormai alla cultural memory nella narrativa contemporanea, cioè alla rappresentazione della migrazione, del Sessantotto e del G8 2001 svoltosi a Genova. Ultimamente si occupa anche di altre espressioni grafiche e dei movimenti antimafia. È editore di 'Moving Texts/Testi mobili', una 'Peter Lang Series'.

KU Leuven - Campus Antwerpen

Sint-Andriesstraat 2

2000 Antwerpen (Belgio)

inge.lanslots@kuleuven.be

Paul Sambre è docente di studi sul discorso e di linguistica italiana presso l'Università di Lovanio (KU Leuven). È membro del gruppo di ricerca MIDI, Multimodalità e Interazione nel Discorso. La sua ricerca è incentrata sulla multimodalità all'intersezione delle tradizioni socio-cognitive, socio-semiotiche e critiche degli studi sul discorso. Questi ultimi anni nella sua attività di ricerca sta affrontando nuovi temi multimodali quali la nozione di embodiment nelle interazioni musicali, la rappresentazione mediatica delle mafie italiane all'estero, e della 'ndrangheta globale nei videoservizi, così come il discorso contro-egemonico e multimodale dei movimenti antimafia italiani. Ha pubblicato su Cognitive Semiotics, Journal of Pragmatics, Incontri: Rivista europea di studi italiani, I-LanD Journal ed è membro del comitato di redazione di Constructions and Frames, ITL -International Journal of Applied Linguistics e I-LanD Journal on Identity, Language and Diversity.

\section{KU Leuven - Campus Antwerpen \\ Sint-Andriesstraat 2 \\ 2000 Antwerpen (Belgio) \\ paul.sambre@kuleuven.be}

Eliana Maestri è Senior Lecturer in Translation Studies and Direttrice del Centro Translating Cultures, presso l'Università Exeter. La sua ricerca si concentra sulle connessioni tra traduzione, cultura visiva e migrazione. Nel 2019, Maestri ha ottenuto fondi dal British Academy Rising Star Engagement Award per organizzare un Festival di Traduzione a Exeter. Nel 2011-12, Maestri ha completato un progetto di post-dottorato negli studi europei, presso l'Università di Sydney, vincendo fondi elargiti dall'EUOSSIC Erasmus Mundus, per poi vincere nel 2014 ulteriori prestigiosi fondi di ricerca presso l'Università di Monash, Melbourne, Australia. Le sue pubblicazioni includono lo studio delle percezioni e visioni europee da parte di gruppi migranti in Australia, le traduzioni di tradizioni mobili nell'ambito della musica folk di origine italiana e australiana (con Rita Wilson) e l'esplorazione multimodale dell'arte visiva prodotta da Jon Cattapan, eminente artista italiano e australiano operante nell'emisfero australe (quest'ultimo studio è contenuto all'interno del volume Transcultural Italies: Mobility, Memory and Translation uscito nel 2020 e curato da Loredana Polezzi, Charles Burdett e Barbara 
Spadaro,). Le pubblicazioni precedenti di Maestri includono la monografia dal titolo Translating the Female Self across Cultures pubblicata nella collana John Benjamins: Translation Library (BTL) nel 2018 e relativa allostudio di traduzioni francesi e italiane di opere autobiografiche realizzate da scrittrici contemporanee in lingua inglese.

University of Exeter

Streatham Campus

Exeter EX4 4PY (Regno Unito)

E.MAESTRI@exeter.ac.uk

\section{SUMMARY}

\section{The commemoration of victims of violence}

Street art, activists and their messages of positivity

The present article analyses how street art and activism intertwine in public spaces addressing wider audiences with messages of growing positivity. Via the comparative analysis of two case studies revolving around the 'no mafia' sign on the outskirts of Palermo (Capaci) and the mural dedicated to Carlo Giuliani at the entrance of the Garbatella area within the Ostiense quarter in Rome, we explore the genesis of both works, their specific spatial setting and the role of social actors in the conservation of the mural paintings, as well as in the commemoration of victims of violence. Our analysis draws on key theoretical findings conceptualized within memory studies, sociology and research on visual culture. 